\title{
Probing the ATIC peak in the cosmic-ray electron spectrum with H.E.S.S.
}

F. Aharonian ${ }^{1,13}$, A. G. Akhperjanian ${ }^{2}$, G. Anton ${ }^{16}$, U. Barres de Almeida ${ }^{8, \star}$, A. R. Bazer-Bachi ${ }^{3}$, Y. Becherini ${ }^{12}$, B. Behera ${ }^{14}$, K. Bernlöhrr ${ }^{1,5}$, A. Bochow ${ }^{1}$, C. Boisson ${ }^{6}$, J. Bolmont ${ }^{19}$, V. Borrel ${ }^{3}$, J. Brucker ${ }^{16}$, F. Brun ${ }^{19}$, P. Brun ${ }^{7}$, R. Bühler ${ }^{1}$, T. Bulik ${ }^{24}$, I. Büsching ${ }^{9}$, T. Boutelier ${ }^{17}$, P. M. Chadwick ${ }^{8}$, A. Charbonnier ${ }^{19}$, R. C. G. Chaves ${ }^{1}$,

A. Cheesebrough ${ }^{8}$, L.-M. Chounet ${ }^{10}$, A. C. Clapson ${ }^{1}$, G. Coignet $^{11}$, M. Dalton ${ }^{5}$, M. K. Daniel ${ }^{8}$, I. D. Davids $^{22,9}$, B. Degrange ${ }^{10}$, C. Deil $^{1}$, H. J. Dickinson ${ }^{8}$, A. Djannati-Ataï ${ }^{12}$, W. Domainko ${ }^{1}$, L. O'C. Drury ${ }^{13}$, F. Dubois ${ }^{11}$, G. Dubus $^{17}$, J. Dyks ${ }^{24}$, M. Dyrda ${ }^{28}$, K. Egberts ${ }^{1, \star \star}$, D. Emmanoulopoulos ${ }^{14}$, P. Espigat ${ }^{12}$, C. Farnier ${ }^{15}$, F. Feinstein ${ }^{15}$, A. Fiasson ${ }^{11}$, A. Förster ${ }^{1}$, G. Fontaine ${ }^{10}$, M. Füßling ${ }^{5}$, S. Gabici ${ }^{13}$, Y. A. Gallant ${ }^{15}$, L. Gérard ${ }^{12}$, D. Gerbig ${ }^{21}$, B. Giebels ${ }^{10}$, J. F. Glicenstein ${ }^{7}$, B. Glück ${ }^{16}$, P. Goret ${ }^{7}$, D. Göring ${ }^{16}$, D. Hauser ${ }^{14}$, M. Hauser ${ }^{14}$, S. Heinz $^{16}$, G. Heinzelmann ${ }^{4}$, G. Henri ${ }^{17}$, G. Hermann ${ }^{1}$, J. A. Hinton ${ }^{25}$, A. Hoffmann ${ }^{18}$, W. Hofmann ${ }^{1, \star \star \star}$, M. Holleran ${ }^{9}$, S. Hoppe ${ }^{1}$, D. Horns ${ }^{4}$, A. Jacholkowska ${ }^{19}$, O. C. de Jager ${ }^{9}$, C. Jahn ${ }^{16}$, I. Jung ${ }^{16}$, K. Katarzyński ${ }^{27}$, U. Katz ${ }^{16}$, S. Kaufmann ${ }^{14}$, E. Kendziorra ${ }^{18}$, M. Kerschhaggl ${ }^{5}$, D. Khangulyan ${ }^{1}$, B. Khélifi ${ }^{10}$, D. Keogh ${ }^{8}$, W. Kluźniak ${ }^{24}$,

T. Kneiske ${ }^{4}$, Nu. Komin ${ }^{15}$, K. Kosack ${ }^{1}$, R. Kossakowski ${ }^{11}$, G. Lamanna ${ }^{11}$, J.-P. Lenain ${ }^{6}$, T. Lohse ${ }^{5}$, V. Marandon ${ }^{12}$, J. M. Martin 6 , O. Martineau-Huynh ${ }^{19}$, A. Marcowith ${ }^{15}$, J. Masbou ${ }^{11}$, D. Maurin ${ }^{19}$, T. J. L. McComb ${ }^{8}$, M. C. Medina ${ }^{6}$, R. Moderski ${ }^{24}$, E. Moulin ${ }^{7}$, M. Naumann-Godo ${ }^{10}$, M. de Naurois ${ }^{19}$, D. Nedbal ${ }^{20}$, D. Nekrassov ${ }^{1}$, B. Nicholas ${ }^{26}$, J. Niemiec ${ }^{28}$, S. J. Nolan ${ }^{8}$, S. Ohm ${ }^{1}$, J.-F. Olive ${ }^{3}$, E. de Oña Wilhelmi ${ }^{1,12,29}$, K. J. Orford ${ }^{8}$, M. Ostrowski ${ }^{23}$, M. Panter ${ }^{1}$, M. Paz Arribas ${ }^{5}$, G. Pedaletti ${ }^{14}$, G. Pelletier ${ }^{17}$, P.-O. Petrucci ${ }^{17}$, S. Pita ${ }^{12}$, G. Puihlhofer ${ }^{14}$, M. Punch ${ }^{12}$,

A. Quirrenbach ${ }^{14}$, B. C. Raubenheimer', M. Raue ${ }^{1,29}$, S. M. Rayner ${ }^{8}$, O. Reimer ${ }^{30}$, M. Renaud ${ }^{1}$, F. Rieger ${ }^{1,29}$, J. Ripken ${ }^{4}$, L. Rob ${ }^{20}$, S. Rosier-Lees ${ }^{11}$, G. Rowell ${ }^{26}$, B. Rudak ${ }^{24}$, C. B. Rulten ${ }^{8}$, J. Ruppel ${ }^{21}$, V. Sahakian ${ }^{2}$, A. Santangelo ${ }^{18}$, R. Schlickeiser ${ }^{21}$, F. M. Schöck ${ }^{16}$, R. Schröder ${ }^{21}$, U. Schwanke ${ }^{5}$, S. Schwarzburg ${ }^{18}$, S. Schwemmer ${ }^{14}$, A. Shalchi ${ }^{21}$, M. Sikora ${ }^{24}$, J. L. Skilton ${ }^{25}$, H. Sol ${ }^{6}$, D. Spangler ${ }^{8}$, E. Stawarz ${ }^{23}$, R. Steenkamp ${ }^{22}$, C. Stegmann $^{16}$, F. Stinzing ${ }^{16}$, G. Superina ${ }^{10}$, A. Szostek ${ }^{23,17}$, P. H. Tam ${ }^{14}$, J.-P. Tavernet ${ }^{19}$, R. Terrier ${ }^{12}$, O. Tibolla ${ }^{1}$, M. Tluczykont ${ }^{4}$, C. van Eldik $^{1}$, G. Vasileiadis ${ }^{15}$, C. Venter ${ }^{9}$, L. Venter ${ }^{6}$, J. P. Vialle ${ }^{11}$, P. Vincent ${ }^{19}$, M. Vivier ${ }^{7}$, H. J. Völk ${ }^{1}$, F. Volpe ${ }^{1}$, S. J. Wagner ${ }^{14}$, M. Ward ${ }^{8}$, A. A. Zdziarski ${ }^{24}$, and A. Zech 6

(Affiliations can be found after the references)

Received 19 September 2009 / Accepted 18 October 2009

\section{ABSTRACT}

The measurement of an excess in the cosmic-ray electron spectrum between 300 and $800 \mathrm{GeV}$ by the ATIC experiment has - together with the PAMELA detection of a rise in the positron fraction up to $\approx 100 \mathrm{GeV}$ - motivated many interpretations in terms of dark matter scenarios; alternative explanations assume a nearby electron source like a pulsar or supernova remnant. Here we present a measurement of the cosmic-ray electron spectrum with H.E.S.S. starting at $340 \mathrm{GeV}$. While the overall electron flux measured by H.E.S.S. is consistent with the ATIC data within statistical and systematic errors, the H.E.S.S. data exclude a pronounced peak in the electron spectrum as suggested for interpretation by ATIC. The H.E.S.S. data follow a power-law spectrum with spectral index of $3.0 \pm 0.1$ (stat.) \pm 0.3 (syst.), which steepens at about $1 \mathrm{TeV}$.

Key words. cosmic rays - methods: data analysis

\section{Introduction}

Very-high-energy $(E \gtrsim 100 \mathrm{GeV})$ cosmic-ray electrons ${ }^{1}$ lose their energy rapidly via inverse Compton scattering and synchrotron radiation resulting in short cooling time and hence range. Therefore, they must come from a few nearby sources (Shen 1970; Aharonian et al. 1995; Kobayashi et al. 2004).

\footnotetext{
* Supported by CAPES Foundation, Ministry of Education of Brazil.

$\star \star$ e-mail: Kathrin.Egberts@mpi-hd.mpg.de

$\star \star \star$ e-mail: Werner.Hofmann@mpi-hd.mpg.de

1 The term electrons is used generically in the following to refer to both electrons and positrons since most experiments do not discriminate between particle and antiparticle.
}

Recently, the ATIC collaboration reported the measurement of an excess in the electron spectrum (Chang et al. 2008). The excess appears as a peak in $E^{3} \Phi(E)$ where $\Phi$ is the differential electron flux; it can be approximated as a component with a power law index around 2 and a sharp cutoff around $620 \mathrm{GeV}$. Combined with the excess in the positron fraction measured by PAMELA (Adriani et al. 2009), the peak feature of the ATIC measurement has been interpreted in terms of a dark matter signal or a contribution of a nearby pulsar (e.g. Malyshev et al. 2009 , and references given there). In the case of dark matter, the structure in the electron spectrum can be explained as caused by dark matter annihilation into low multiplicity final states, while in the case of a pulsar scenario the structure arises from a 
competition between energy loss processes of pulsar electrons (which impose an energy cutoff depending on pulsar age) and energy-dependent diffusion (which favors high-energy particles in case of more distant pulsars).

The possibility to distinguish between a nearby electron source and a dark matter explanation with imaging atmospheric Cherenkov telescopes has been discussed by Hall \& Hooper (2009). Imaging atmospheric Cherenkov telescopes have five orders of magnitude larger collection areas than balloon and satellite experiments and can therefore measure $\mathrm{TeV}$ electrons with excellent statistics. Hall and Hooper assume that a structure in the electron spectrum should be visible even in the presence of a strong background of misidentified nucleonic cosmic rays. However, the assumption of a smooth background is oversimplified; in typical analyses the background rejection varies strongly with energy and without reliable control or better subtraction of the background, decisive results are difficult to achieve. In a recent publication, the High Energy Stereoscopic System (H.E.S.S.) collaboration has shown that such a subtraction is indeed possible, reporting a measurement of the electron spectrum in the range of $700 \mathrm{GeV}$ to $5 \mathrm{TeV}$ (Aharonian et al. 2008).

\section{The low-energy extension of the H.E.S.S. electron measurement}

Here an extension of the H.E.S.S. measurement towards lower energies is presented, partially covering the range of the reported ATIC excess. H.E.S.S. (Hinton 2004) is a system of four imaging atmospheric Cherenkov telescopes in Namibia. While designed for the measurement of $\gamma$-ray initiated air-showers, it can be used to measure cosmic-ray electrons as well. The basic properties of the analysis of cosmic-ray electrons with H.E.S.S. have been presented in Aharonian et al. (2008). For the analysis, data from extragalactic fields (with a minimum of $7^{\circ}$ above or below the Galactic plane) are used excluding any known or potential $\gamma$-ray source in order to avoid an almost indistinguishable $\gamma$-ray contribution to the electron signal. As the diffuse extragalactic $\gamma$-ray background is strongly suppressed by pair creation on cosmic radiation fields (Coppi \& Aharonian 1997), its contribution to the measured flux can be estimated following Coppi \& Aharonian (1997) to be less than 6\%, assuming a blazar spectrum of an unbroken powerlaw up to $3 \mathrm{TeV}$ with a Gaussian spectral index distribution centered at $\Gamma=-2.1$ with $\sigma_{\Gamma}=0.35$. For an improved rejection of the hadronic background a Random Forest algorithm (Breiman \& Cutler 2004) is used. The algorithm uses image information to estimate the electron likeness $\zeta$ of each event. Since some of the image parameters used to derive the $\zeta$ parameter are energy dependent, also $\zeta$ depends on energy. To derive an electron spectrum, a cut on $\zeta$ of $\zeta>0.6$ is applied and the number of electrons is determined in independent energy bands by a fit of the distribution in $\zeta$ with contributions of simulated electrons and protons. The contribution of heavier nuclei is sufficiently suppressed for $\zeta>0.6$ as not to play a role. The result does not depend on the particular choice of $\zeta_{\min }$. For an extension of the spectrum towards lower energies, the analysis has been modified to improve the sensitivity at low energies. In the event selection cuts, the minimum image amplitude has been reduced from 200 to 80 photo electrons to allow for lower energy events. In order to guarantee good shower reconstruction, only events with a reconstructed distance from the projected core position on the ground to the array center of less than $100 \mathrm{~m}$ are included. Additionally, only data taken between 2004 and

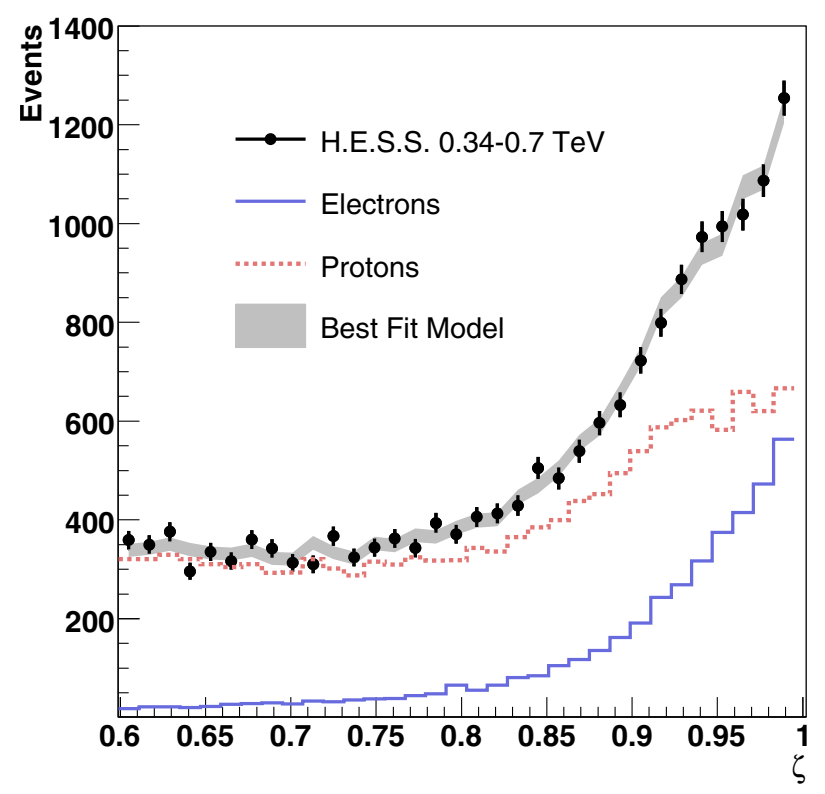

Fig. 1. The measured distribution of the parameter $\zeta$, compared with distributions for simulated protons and electrons, for showers with reconstructed energy between 0.34 and $0.7 \mathrm{TeV}$ (the energy range of the extension towards lower energies compared to the analysis presented in Aharonian et al. 2008). The best fit model combination of electrons and protons is shown as a shaded band. The proton simulations use the SIBYLL hadronic interaction model. Distributions differ from the ones presented in Fig. 1 of Aharonian et al. (2008) because of the energy dependence of the $\zeta$ parameter.

2005 are used. The reason is that the H.E.S.S. mirror reflectivity degrades over time and a reduced light yield corresponds to an increased energy threshold. The new data and event selection reduces the event statistics but enables to lower the analysis threshold to $340 \mathrm{GeV}$. The effective collection area at $340 \mathrm{GeV}$ is $\approx 4 \times 10^{4} \mathrm{~m}^{2}$. With a live-time of $77 \mathrm{~h}$ of good quality data, a total effective exposure of $\approx 2.2 \times 10^{7} \mathrm{~m}^{2} \mathrm{sr}$ is achieved at $340 \mathrm{GeV}$. Owing to the steepness of the electron spectrum, the measurement at lower energies is facilitated by the comparatively higher fluxes. The $\zeta$ distribution in the energy range of 340 to $700 \mathrm{GeV}$ is shown in Fig. 1.

The low-energy electron spectrum resulting from this analysis is shown in Fig. 2 together with previous data of H.E.S.S. and direct measurements. The spectrum is well described by a broken power law $\mathrm{d} N / \mathrm{d} E=k \cdot\left(E / E_{\mathrm{b}}\right)^{-\Gamma_{1}} \cdot\left(1+\left(E / E_{\mathrm{b}}\right)^{1 / \alpha}\right)^{-\left(\Gamma_{2}-\Gamma_{1}\right) \alpha}$ $\left(\chi^{2} /\right.$ d.o.f. $\left.=5.6 / 4, p=0.23\right)$ with a normalization $k=$ $(1.5 \pm 0.1) \times 10^{-4} \mathrm{TeV}^{-1} \mathrm{~m}^{-2} \mathrm{sr}^{-1} \mathrm{~s}^{-1}$, and a break energy $E_{\mathrm{b}}=$ $0.9 \pm 0.1 \mathrm{TeV}$, where the transition between the two spectral indices $\Gamma_{1}=3.0 \pm 0.1$ and $\Gamma_{2}=4.1 \pm 0.3$ occurs. The parameter $\alpha$ denotes the sharpness of the transition, the fit prefers a sharp transition, $\alpha<0.3$. The shaded band indicates the uncertainties in the flux normalization that arise from uncertainties in the modeling of hadronic interactions and in the atmospheric model. The uncertainties amount to about $30 \%$ and are derived in the same fashion as in the initial paper (Aharonian et al. 2008), i.e. by comparison of the spectra derived from two independent data sets taken in summer and autumn 2004 for the effect of atmospheric variations and by comparison of the spectra derived using the SIBYLL and QGSJET-II hadronic interaction model for the effect of the uncertainties in the proton simulations. The band is centered around the broken power law fit. The systematic error on the spectral indices $\Gamma_{1}, \Gamma_{2}$ is $\Delta \Gamma$ (syst.) $\lesssim 0.3$. The H.E.S.S. energy scale uncertainty of $15 \%$ is visualized by the double arrow. 


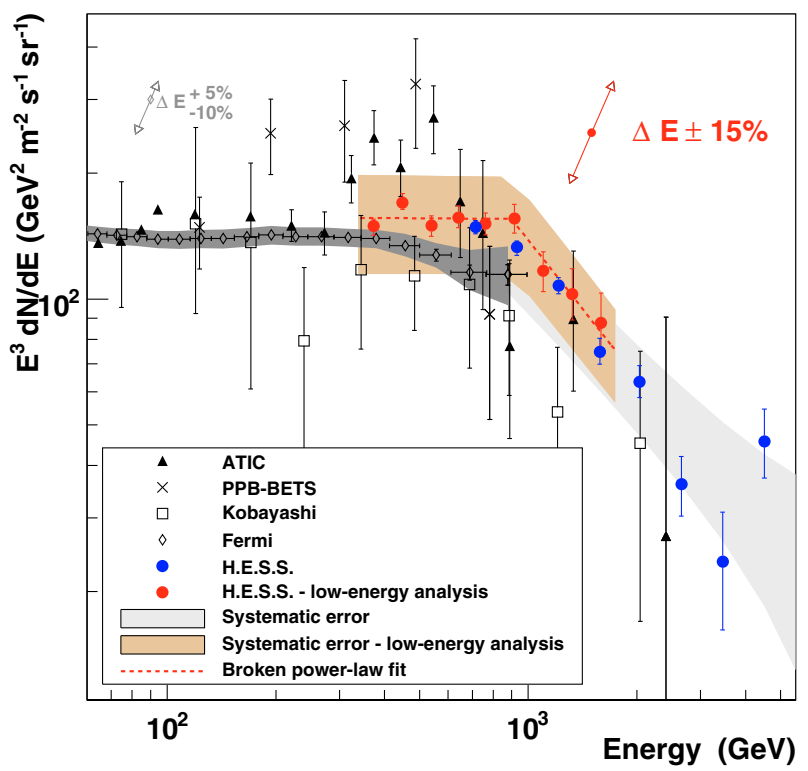

Fig. 2. The energy spectrum $E^{3} \mathrm{~d} N / \mathrm{d} E$ of cosmic-ray electrons as measured by ATIC (Chang et al. 2008), PPB-BETS (Torii et al. 2008), emulsion chamber experiments (Kobayashi et al. 2004), FERMI (Abdo et al. 2009) (the gray band shows the FERMI systematic uncertainty, the double arrow labeled with ${ }_{-10 \%}^{+5 \%}$ the uncertainty of the FERMI energy scale), and H.E.S.S. Previous H.E.S.S. data (Aharonian et al. 2008) are shown as blue points, the result of the low-energy analysis presented here as red points. The shaded bands indicate the approximate systematic error arising from uncertainties in the modeling of hadronic interactions and in the atmospheric model in the two analyses. The double arrow indicates the effect of an energy scale shift of $15 \%$, the approximate systematic uncertainty on the H.E.S.S. energy scale. The fit function is described in the text.

\section{Interpretation}

The H.E.S.S. measurement yields a smooth spectrum with a steepening towards higher energies, confirming the earlier findings above $600 \mathrm{GeV}$ (Aharonian et al. 2008).

When compared to ATIC, the H.E.S.S. data show no indication of an excess and sharp cutoff in the electron spectrum as reported by the ATIC collaboration. Since H.E.S.S. measures the electron spectrum only above $340 \mathrm{GeV}$, one cannot test the rising section of the ATIC-reported excess. Although different in shape, an overall consistency of the ATIC spectrum with the H.E.S.S. result can be obtained within the uncertainty of the H.E.S.S. energy scale of about $15 \%$. The deviation between the ATIC and the H.E.S.S. data is minimal at the $20 \%$ confidence level (assuming Gaussian errors for the systematic uncertainty dominating the H.E.S.S. measurement) when applying an upward shift of $10 \%$ in energy to the H.E.S.S. data. The shift is well within the uncertainty of the H.E.S.S. energy scale. In this case the H.E.S.S. data overshoot the measurement of balloon experiments above $800 \mathrm{GeV}$, but are consistent given the large statistical errors from balloon experiments at these energies. However, the nominal H.E.S.S. data are in very good agreement with the high precision FERMI measurement up to $1 \mathrm{TeV}$. The combined H.E.S.S. and FERMI measurements make a feature in the electron spectrum in the region of overlap of both experiments rather unlikely.

Beside comparing the H.E.S.S. measurement with ATIC and FERMI data, we also put the Kaluza-Klein (KK) interpretation suggested by Chang et al. (2008) to test: A model calculation of how the therein proposed $\mathrm{KK}$ particle with a mass of $620 \mathrm{GeV}$

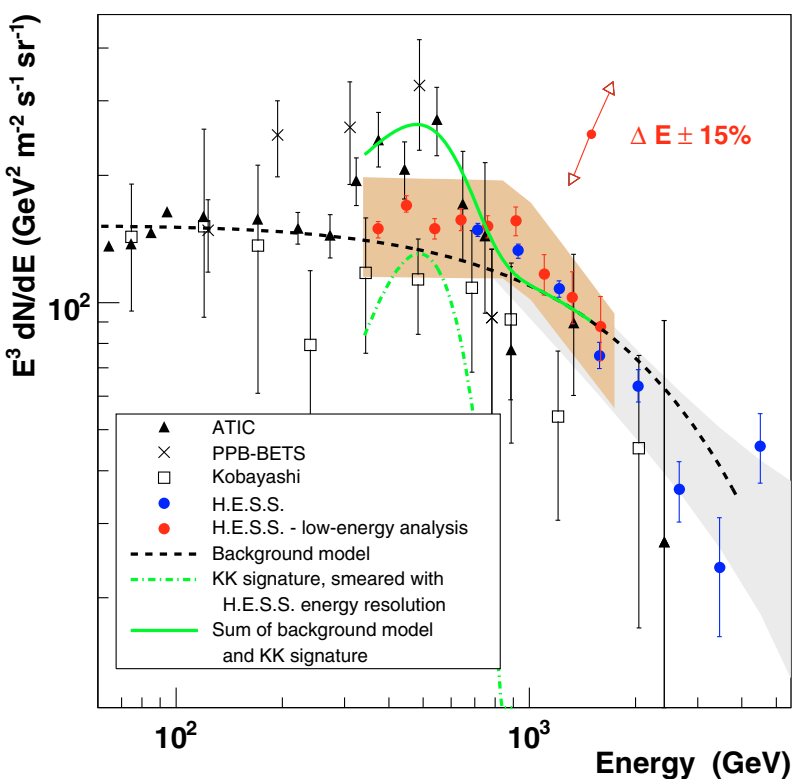

Fig. 3. The energy spectrum $E^{3} \mathrm{~d} N / \mathrm{d} E$ of cosmic-ray electrons measured by H.E.S.S. and balloon experiments. Also shown are calculations for a Kaluza-Klein signature in the H.E.S.S. data with a mass of $620 \mathrm{GeV}$ and a flux as determined from the ATIC data (dashed-dotted line), the background model fitted to low-energy ATIC and high-energy H.E.S.S. data (dashed line) and the sum of the two contributions (solid line). The shaded regions represent the approximate systematic error as in Fig. 2.

and a flux approximated to fit the ATIC data would appear in the H.E.S.S. data is shown in Fig. 3. Here electron air showers are simulated with an energy distribution following the energy spectrum of the KK signature presented by the ATIC collaboration. The simulated events and their energy are reconstructed by the H.E.S.S. data analysis. With the use of the effective collection area and the "observation time" that the number of simulations corresponds to, the KK spectrum is obtained as it would be resolved by H.E.S.S. Due to the limited energy resolution of about $15 \%$, a sharp cutoff at the energy of the KK mass would have been smeared out. The residual background spectrum to a $\mathrm{KK}$ signal is modeled by a power law with exponential cutoff, which is fitted to the low-energy ATIC data $(E<300 \mathrm{GeV})$ and the high-energy H.E.S.S. data $(E>700 \mathrm{GeV})$. Accordingly, our background spectrum deviates from the GALPROP prediction as used in Chang et al. (2008). Fixing the background spectrum to most recent observational data is preferable since the Galactic electron spectrum at highest energies might carry the signature of nearby electron sources (Pohl \& Esposito 1998) and can therefore differ substantially from the model calculation. The sum of the KK signal and electron background spectrum above $340 \mathrm{GeV}$ is shown as solid curve in Fig. 3. The shape of the predicted spectrum for the case of a KK signal is not compatible with the H.E.S.S. data at the $99 \%$ confidence level.

Despite superior statistics, the H.E.S.S. data do not rule out the existence of the ATIC-reported excess owing to a possible energy scale shift inherent to the presented measurement. Whereas compatibility with FERMI and ATIC data is confirmed, the KK scenario of Chang et al. (2008) cannot be easily reconciled with the H.E.S.S. measurement. The spectrum rather exhibits a steepening towards higher energies and is therefore compatible with conventional electron populations of astrophysical origin within the uncertainties related to the injection spectra and propagation effects. 
Acknowledgements. The support of the Namibian authorities and of the University of Namibia in facilitating the construction and operation of H.E.S.S. is gratefully acknowledged, as is the support by the German Ministry for Education and Research (BMBF), the Max Planck Society, the French Ministry for Research, the CNRS-IN2P3 and the Astroparticle Interdisciplinary Programme of the CNRS, the U.K. Science and Technology Facilities Council (STFC), the IPNP of the Charles University, the Polish Ministry of Science and Higher Education, the South African Department of Science and Technology and National Research Foundation, and by the University of Namibia. We appreciate the excellent work of the technical support staff in Berlin, Durham, Hamburg, Heidelberg, Palaiseau, Paris, Saclay, and in Namibia in the construction and operation of the equipment.

\section{References}

Abdo, A. A., Ackermann, M, Ajello, M., et al. 2009, Phys. Rev. Lett., 102, 181101

Adriani, O., Barbarino, G. C., Bazilevskaya, G. A., et al. 2009, Nature, 458, 607

Aharonian, F. A., Atoyan, A. M., \& Völk, H. J. 1995, A\&A, 294, L41

Aharonian, F. A., et al. 2008, Phys. Rev. Lett., 101

Breiman, L., \& Cutler, A. 2004,

http: //www.stat. berkeley.edu/ breiman/RandomForests/

Chang, J., Adams, J. H., Ahn, H. S., et al. 2008, Nature, 456, 362

Coppi, P., \& Aharonian, F. A. 1997, ApJ, 487, L9

Hall, J., \& Hooper, D. 2009, Phys. Lett. B, 681, 220

Hinton, J. A. H. C. 2004, New Astron. Rev., 48, 331

Kobayashi, T., Komori, Y., Yoshida, K., \& Nishimura, J. 2004, ApJ, 601, 340

Malyshev, D., Cholis, I., \& Gelfand, J. 2009, Phys. Rev. D, 80

Pohl, M., \& Esposito, J. A. 1998, ApJ, 507, 327

Shen, C. S. 1970, ApJ, 162, L181

Torii, S., et al. 2008, Astropart. Phys. [astro-ph/0809.0760]

1 Max-Planck-Institut für Kernphysik, PO Box 103980, 69029 Heidelberg, Germany

e-mail: kathrin.Egberts@uibk . ac . at

2 Yerevan Physics Institute, 2 Alikhanian Brothers St., 375036 Yerevan, Armenia

3 Centre d'Étude Spatiale des Rayonnements, CNRS/UPS, 9 Av. du Colonel Roche, BP 4346, 31029 Toulouse Cedex 4, France

4 Universität Hamburg, Institut für Experimentalphysik, Luruper Chaussee 149, 22761 Hamburg, Germany

5 Institut für Physik, Humboldt-Universität zu Berlin, Newtonstr. 15, 12489 Berlin, Germany

6 LUTH, Observatoire de Paris, CNRS, Université Paris Diderot, 5 place Jules Janssen, 92190 Meudon, France

7 IRFU/DSM/CEA, CE Saclay, 91191 Gif-sur-Yvette, Cedex, France

8 University of Durham, Department of Physics, South Road, Durham DH1 3LE, UK
9 Unit for Space Physics, North-West University, Potchefstroom 2520, South Africa

10 Laboratoire Leprince-Ringuet, École Polytechnique, CNRS/IN2P3, 91128 Palaiseau, France

11 Laboratoire d'Annecy-le-Vieux de Physique des Particules, Université de Savoie, CNRS/IN2P3, 9 chemin de Bellevue, BP 110, 74941 Annecy-le-Vieux Cedex, France

12 Astroparticule et Cosmologie (APC), CNRS, Université Paris 7 Denis Diderot, 10 rue Alice Domon et Léonie Duquet, 75205 Paris Cedex 13, France, UMR 7164 (CNRS, Université Paris VII, CEA, Observatoire de Paris)

13 Dublin Institute for Advanced Studies, 5 Merrion Square, Dublin 2, Ireland

14 Landessternwarte, Universität Heidelberg, Königstuhl, 69117 Heidelberg, Germany

15 Laboratoire de Physique Théorique et Astroparticules, Université Montpellier 2, CNRS/IN2P3, CC 70, Place Eugène Bataillon, 34095 Montpellier Cedex 5, France

16 Universität Erlangen-Nürnberg, Physikalisches Institut, ErwinRommel-Str. 1, 91058 Erlangen, Germany

17 Laboratoire d'Astrophysique de Grenoble, INSU/CNRS, Université Joseph Fourier, BP 53, 38041 Grenoble Cedex 9, France

18 Institut für Astronomie und Astrophysik, Universität Tübingen, Sand 1, 72076 Tübingen, Germany

19 LPNHE, Université Pierre et Marie Curie Paris 6, Université Denis Diderot Paris 7, CNRS/IN2P3, 4 place Jussieu, 75252 Paris Cedex 5, France

20 Charles University, Faculty of Mathematics and Physics, Institute of Particle and Nuclear Physics, V Holešovičkách 2, 18000 Prague 8, Czech Republic

21 Institut für Theoretische Physik, Lehrstuhl IV: Weltraum und Astrophysik, Ruhr-Universität Bochum, 44780 Bochum, Germany

22 University of Namibia, Private Bag 13301, Windhoek, Namibia

23 Obserwatorium Astronomiczne, Uniwersytet Jagielloński, Kraków, Poland

24 Nicolaus Copernicus Astronomical Center, ul. Bartycka 18, 00-716 Warsaw, Poland

25 School of Physics \& Astronomy, University of Leeds, Leeds LS2 9JT, UK

26 School of Chemistry \& Physics, University of Adelaide, Adelaide 5005, Australia

27 Toruń Centre for Astronomy, Nicolaus Copernicus University, ul. Gagarina 11, 87-100 Toruń, Poland

28 Instytut Fizyki Ja̧drowej PAN, ul. Radzikowskiego 152, 31-342 Kraków, Poland

29 European Associated Laboratory for Gamma-Ray Astronomy, jointly supported by CNRS and MPG

30 Stanford University, HEPL \& KIPAC, Stanford, CA 94305-4085, USA 